\title{
Anusual Application of Partial Discharges to Diagnose of High Voltage Power Transformers
}

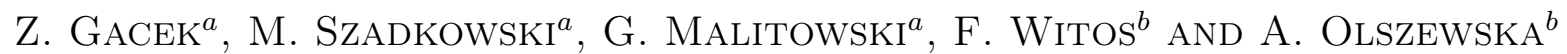 \\ ${ }^{a}$ Institute of Power System and Control, Silesian University of Technology \\ B. Krzywoustego 2, 44-100 Gliwice, Poland \\ ${ }^{b}$ Department of Optoelectronics, Silesian University of Technology \\ B. Krzywoustego 2, 44-100 Gliwice, Poland
}

\begin{abstract}
The article contains information on research carried out in Electric Faculty of Silesian University of Technology, made at test stations and aimed at diagnosis how is actual technical state of high voltage power oil transformers. Opinion on a state of transformers is based on analysis results of partial discharge measurements. Authors use simultaneously: electric, acoustic and dissolved gas analysis method. Applied acoustic method is the original own one. Non-conventional application of electric method is proposed, too. In the frame of chosen methodology the partial discharges are measured and next calculations of quantities describing partial discharges within particular methods are carried out in order to describe phenomena commonly for electric and acoustic method. Information sources about level and kind of partial discharges are measurement results obtained from electric method whereas information on places where partial discharges appear is contained in measurement results coming from acoustic method. Authors present selected application results of such a combined measuring method.
\end{abstract}

PACS: 43.40.Le, 52.80.s, 07.50.Hp

\section{Introduction}

Since many years, diagnosis of power transformers being in operation becomes more and more important for their users. It makes up many factors but the most important are: growing numbers of transformers which exceed time period of their operation ( 25 to 30 years) anticipated by constructors, and introduction of "economies" within design and construction of insulation systems resulting for new transformers with a bigger tendency to failures. It is important that each failure of a transformer is connected not only with serious financial wastes and challenge of trust to its owner but may cause threat of human life and environmental contamination. Despite, many of transformer owners aim at moving away in time exchange of long-serving unities into new ones. Besides, when such an exchange is carried out, the owner wants to know how is the level of "certainty" so that new transformer works correctly. Trustworthy but not influencing onto operation diagnostic methods are searched in these two situations. A rule in many countries becomes execution of periodical control measurements and accumulation of operation documentation of particular transformers $[1,2]$. This task is entrusted to companies who specialize in such diagnostic tests. These companies are obliged to warn the power operator about necessity of renovation or exchange of the unity endangered by failure. Such a dealing is helpful when the state of transformer insulation system deteriorates slowly, which con- cerns many numbers of analyzed failures of transformers. There are also failures which develop very quickly - during some weeks, days and even hours. One way to predict such events is a diagnosis realized on-line.

There are many diagnostic methods used both in periodic and on-line evaluation of transformers. Unfortunately, majority of these methods gives only a partial knowledge on the state of a transformer, principally on the state of its insulation systems. Methods of partial discharges measurements belong to the group which serve to selectively detect and to locate the places threatened by puncture of any from insulation systems.

Methods of acoustic emission (AE) applied nowadays in order to detect partial discharges (PD) within transformers during their operation are more and more effective. They enable us to find out existence of partial discharge sources and location of them. A problem is still evaluation of menace degree of insulation by detected sources. In this case the electric method applied to measure partial discharges becomes very useful. Nowadays, due to lack of low adjustments and recognized methodology how to apply electric method in the case of transformers being in operation, such a method can be applied only at test stations of companies who produce and repair transformers. Authors hope for creation the possibilities so that such a method should be also applied at least in the case of some transformers being in operation. One of considerable conditions relating to correct diagnosis of transformer insulation systems carried out by 
methods basing on measurements of partial discharges is account of different outer noises and their disadvantageous influence on measurement results. One of way to separate outer noises from diagnostic impulses generated by partial discharges in transformer insulation is analysis of correlation degree of electric signals measured in the bushing and acoustic signals measured by means of special sensors (fastened at different places of transformer vat). Assuming that detection of electric signals and non-detection of acoustic signals is caused by an outer noise (example corona discharge), whereas detection of acoustic signals and non-detection of electric signals is caused by sound sources of non-connected with partial discharges (mechanical vibrations, stream of sand grains, rain etc.), then simultaneous application of electric and acoustic method may have the desired result. Taking into account also measurement results of dissolved gas analysis (DGA), such a method can be a base to attempt properly activities connected with the transformer being in diagnosis. Such an idea of diagnosis of power oil transformers is the subject of the article.

\section{Basic methods used to measure of partial discharges in transformer insulation systems}

Partial discharges occurring within insulation systems of high voltage power equipment are one of important information source about technical state of equipment. First noted scientific information relating to PD had place at 1777 year, but dynamic development of these researches began in 20th century and is continued. Relevant work, containing review of methods and systems for PD measurements, comes from 1975 year [1]. As a term "PD evaluation" is understood the following activities: detection, measurement, and location. $\mathrm{Na}$ ture of PD phenomena is admittedly "electric", but it is not change the fact that partial discharges are followed by many other physical phenomena: chemical changes within insulation, stroke elastic deformations and resultant generation of elastic waves of AE as well as thermoemission and photoemission. In order to measure effects of these phenomena, different measuring methods and connected with them ways of PD evaluations are used. Occurrence of current impulses and emission of electromagnetic waves is the basis of electric method of PD measurement, chemical changes of insulating materials are the basis of gaseous chromatography, stroke elastic deformations and accompanied to them emission of acoustic waves are the basis of acoustic method, emission of light radiation is the basis of optical spectrophotometry, local increase of temperature in $\mathrm{PD}$ area is the basis of thermovision method, whereas change of pressure within PD canal is the basis of analysis of these changes. Nowadays, only three first methods of PD evaluation are relevant to practice. In three remaining cases the effects of discharges are very weak and hardly measurable.

Using electric method of PD detection and measurement the following distributions of measured quantities are obtained: phase, amplitude and amplitude--phase distributions [2-5]. Measuring device can be the simplest measuring instrument of apparent charge (ERA devices from F.C. Robinson firm or type of 66/5700 from Haefely Trench firm are the most widespread in Poland), as well as complex wideband measuring systems, for example TE-571 from Haefely Trench firm. TE-571 detector enables us to measure partial discharges in the range of measuring voltage from 40 to $420 \mathrm{~Hz}$. Measuring range of apparent charge locates at interval from zero to $999 \mathrm{nC}$, whereas measuring limits of apparent charge are $40 \mathrm{kHz}$ and $400 \mathrm{kHz}$. In total, there are 23 distributions and time courses describing analyzed kind of PD after measurements $[6,7]$ :

- phase distributions of maximum value of apparent charge, mean value of apparent charge, number of charges, sum of charges;

- distribution of charge intensity depending on apparent charge value and PD energy;

- quantities describing PD in domain of the time: level of maximum value of apparent charge, level of mean value of apparent charge, PD current, maximum value of $\mathrm{PD}$ energy, mean value of $\mathrm{PD}$ energy, power of $\mathrm{PD}$, momentary value of $\mathrm{PD}$ ignition voltage, momentary value of $\mathrm{PD}$ extinguishing voltage, momentary value of $\mathrm{D}$ indicator;

- phase distributions of the following quantities: maximum value of apparent charge, mean value of apparent charge, PD number, sum of charges (also in time function);

- amplitude distributions of intensity and PD energy in time function;

- phase pictures of discharges: 3D and the so-called "Color".

Such a wide description of PD within considered object enables us to determine their kind and physical character. Each of the above-mentioned quantities contains only part of quality and quantity information about partial discharges. The basis for identification of PD occurring within tested insulation system is simultaneous analysis of information coming from particular distributions. DSW (TEAS) software increases capabilities of TE-571 detector. Statistical tools contained within such a program enable us to obtain a fair set of parameters describing tested object. Patterns coming from the library of recognized PD statistical pictures or for example virtual neuron networks enable us to identify better partial discharges. Analysis of possibilities of electric methods leaves to the following conclusion: by means of them location, measurement and - in any cases - location of partial discharges is possible.

$\mathrm{AE}$, understood as phenomenon and measuring method, has been applied for first time in 1965 year [6]. 
This topic is still developed and has more and more importance $[1,8,9]$. PD investigations in Poland by means of acoustic methods can be divided into two stages: the first, resumed by the monograph edited at 1993 year [10], and the second, contemporary, which is based on advanced analysis of $\mathrm{AE}$ signals in domain of the time, frequency, time and frequency and discrimination threshold. One- and many-sources of PD have been modeled in the frame of the first stage. For such models, tested in laboratory, the following descriptors have been analyzed: rate of $\mathrm{AE}$ counts, RMS of a signal, maximum amplitude of a signal, amplitude spectrum of a signal. The movable diagnostic laboratory enables us to diagnose real power transformers. Description of partial discharges applied during such tests consists in choice of discrimination threshold, realization of $\mathrm{AE}$ measurements for a given measuring situation, elaboration of the "map" of rate of AE counts and drawing of conclusions on AE activity. Unfortunately, this research proves that measuring process depends strongly up on inner acoustic and electric noises [11-13]. This was a reason, among other things, that parameters combining partial discharges with basic AE descriptors have been defined unequivocally. Analysis of frequency distributions of AE impulses at the second stage brings about definition of advanced AE descriptors, particularly in the range of dominant frequencies [14]. Descriptor values have been determined for many AE sources and noises accompanied of AE measurements. Analysis of AE signals in domain of threshold discrimination - basing on the shape of amplitude distributions of AE signals coming from different PD sources - was carried out by pattern recognition method basing on neuron networks [6]. Results of this analysis prove in both cases that there are dependences between electric apparent charge, introduced by AE source, and suitable parameter characterizing used method of analysis. Found interdependences are true for measuring situations defined generally by the following conditions: multiple installation but stable location of AE sensor and PD sources. Existence of such correlations enables us to calibrate results obtained by means of $\mathrm{AE}$ method basing on apparent electric charge introduced by tested AE source. Then, there was a new measuring situation, enabling to choose much more objective criteria for evaluation of both activity and location of AE sources within tested transformers. This is the method used by the authors of the article.

Analysis of gaseous products coming from decomposition of oil-paper insulation to diagnose transformers has used since almost 100 years. In the course of this time period the mechanisms of insulation degradation, gases occurring during this process and factors affecting their solubility in the oil, were recognized. Theoretical knowledge, laboratory investigations and operational practice enable to work out numerical dependences between probable defects and concentration of particular gases dissolved into oil. However, these dependences are verified and upgraded continually. Chromatographic analysis of gases dissolved in the oil, called DGA, enables us to detect inner and slowly developed defects - principally discharges with different energy density and overheatings giving a chance to use appropriate measures as well as to evade of expensive disconnections or failures. Thus, nowadays DGA is very efficient method to evaluate technical state of transformers [2, 10, 15].

Framework Instruction of Operation of Transformers, worked out in 2001 year by Energopomiar-Elektryka Gliwice [2] treats chromatographic analysis of gases dissolved into oil as a basic method of operational tests (periodical) and inspections after failures of transformers. DGA method is also applied during reception tests of new transformers as well as modernized unities (with complete exchange of insulation).

From the above information it follows that measurement results coming from each of presented diagnostic methods (electric, acoustic emission and DGA) contain in given conditions only part of information relating to possible defects of composite insulation system in a given device. It is assumed that simultaneous application of electric and acoustic method to analyze partial discharges, supported additionally by DGA method, creates more possibilities to diagnose insulation systems. This is an idea of combined method to diagnose transformers, proposed by the authors, in which simultaneous analysis of diagnostic signals is used at this time independently in electric, acoustic and DGA method.

\section{Combined method of partial discharges measurements}

Combined method of PD measurements means a diagnostic method in the frame of which:

- PD measurements are carried out simultaneously by three methods: electric, acoustic and DGA;

- calculations of measures of quantities describing $\mathrm{PD}$ phenomena in particular methods are made;

- results obtained by three methods are used for reciprocal verification and determination of final description of insulation system state.

Such an approach enables to local and global description of PD phenomena, increases considerably information quality, and simultaneously decreases mistaken interpretation of results obtained only by one measuring method.

From such subject matter the following detailed purposes result:

- recognition of possibility to adapt the integrated method in order to create conception of mobile measuring system;

— working out of algorithm (method) how to proceed when technical state of insulation systems of oil power transformers are valuated basing on three different diagnostic methods; 
- verification what are possibilities so that three methods applied to diagnose of insulation systems within transformers should be used;

- verification of coincidence of diagnostic results obtained from different methods;

— obtainment of complementarity of applied methods in integrated diagnosis of transformers;

- investigation what are possibilities to eliminate mutually disadvantages of particular methods;

- application of wavelet decomposition of AE signals to descript PD phenomena;

- application of the neuron network with supervised learning (for electric and acoustic signals as well as chromatographic data) to describe PD;

- elaboration of a tool for interpretation (expert system) based on three diagnostic methods.

Nowadays, basing on tests of actual objects, there are more and more expanded data base containing test results of actual high voltage transformers investigations and will be continued both in new unities and given to repair.

\section{Chosen measuring results obtained by combined method basing on partial discharges measurements}

Measurement results obtained during tests of two transformers are presented. One of these transformers is a typical unity of $16 \mathrm{MVA}, 110 \mathrm{kV}$, after long period of its operation (marked as T1). The second was new unity of $25 \mathrm{MVA}, 220 \mathrm{kV}$ (marked as T2).

Basing on measurements carried out by means of DGA method, occurrence of partial discharges within insulation system of T1 transformer (Fig. 1) has been stated.

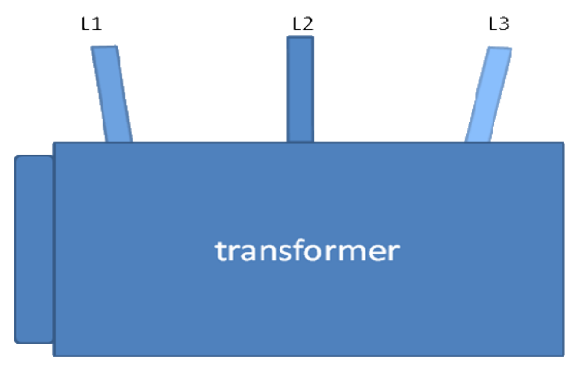

Fig. 1. Sketch of T1 transformer.

In order to confirm existence of partial discharges and to determine their kind, intensity and places when they occur, the combined (electric and acoustic) method was used.

Measurements of PD within transformer insulation systems are made at diagnostic stations by means of electric method according to PN-EN 60270:
- one of transformer phases was energized at LV side (remaining two phases were earthed in the same time);

- in order to avoid corona phenomenon at output of the windows, the screens in bushings situated at $\mathrm{HV}$ side are installed;

- each of phases is tested separately;

- voltage level is regulated according to a suitable graphic in defined time intervals.

In order to simulate conditions at the place of transformer operation, the authors were decided to use a non-conventional way of exercise of measurements:

- all 3 phases of the transformer were energized at LV side;

- screens in the bushings at HV side were not applied;

- partial discharges are measured in each phase separately but the measuring signal contained also PD signals appearing in two remaining phases;

- measurements were carried out in power-frequency voltage (voltage value not greater than nominal value).

As the result of measurements the fair set of parameters characterizing partial discharges within the transformer are obtained. On this base one can find out that there is a very intensive source of inner PD (level of apparent charge approximately $20 \mathrm{nC}$ ) connected with L3 phase (Fig. 1) and one source of inner PD (level of apparent charge approximately $2.5 \mathrm{nC}$ ) connected with L1 phase. A signal generated by the corona at ends of non-screened bushings is recorded simultaneously (Fig. 2). Phase screens $D(q, \varphi, n)$ and phase distributions $D q(\varphi)$ shown in Fig. 2 should be commented.
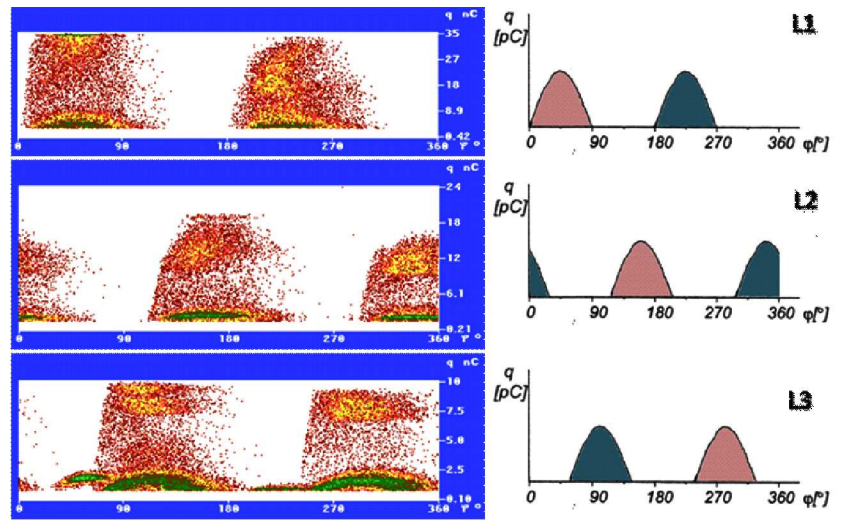

Fig. 2. Phase pictures $D(q, \varphi, n)$ and phase distributions $D q(\varphi)$ of partial discharges in L1, L2 and L3 phase of the tested transformer.

Analysis of PD measuring results for L3 phase of tested transformer proved that the source of partial discharges 
placed inside this phase is connected with L3 phase. A classic for such discharge phase picture of PD intensity was recorded $[5,7]$. Phase range of discharges in the period of the measuring voltage is included within the following brackets: from $0^{\circ}$ to $90^{\circ}$ and from $80^{\circ}$ to $270^{\circ}$; PD intensity is symmetrical within the above brackets. PD distributions and pictures coming from the same source were recorded in L2 and L3 phases. This is testified by the fact that - in comparison with classical phase distribution recorded in L1 phase - phase distribution was displaced by $120^{\circ}$ in L2 phase and by $240^{\circ}$ in L3 phase. Simultaneously, the typical phase picture of other inner source connected with insulating system in L3 phase was recorded. There was interesting a comparison of results coming from acoustic emission method basing on registration of AE signals recorded at several dozen points of lateral walls of the transformer. AE signal were subjected to numerical treatment basing on original method elaborated by Witos, named by its creator as "modified maximum volume method" [6, 16-19]. The result of above activities was maps of ADC descriptors (Fig. 3) for two frequency ranges: $100-200 \mathrm{kHz}$ and $50-100 \mathrm{kHz}$.
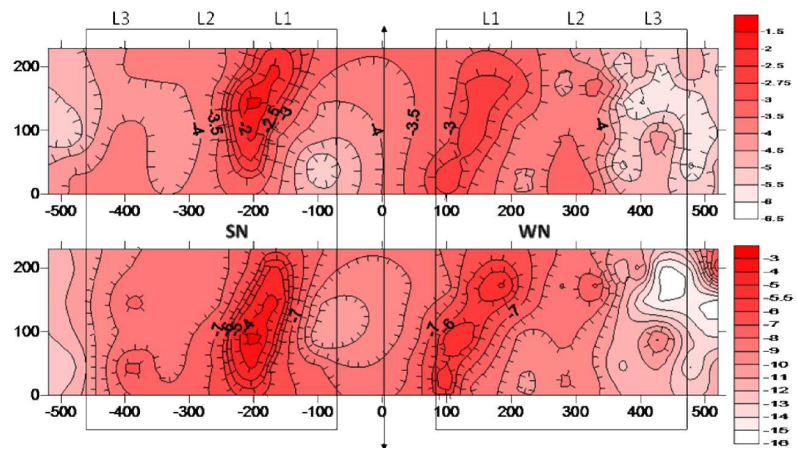

Fig. 3. Maps of ADC descriptors for T1 transformer worked up after analysis of recorded AE signals within the band of $100-200 \mathrm{kHz}$ (upper part) and $50-100 \mathrm{kHz}$ (bottom part).

Descriptor maps are presented in the background of developed lateral walls of the transformer. The wall visible at the left side of Fig. 3 ("negative values" of distances from the center of the tap changer) is the wall of the transformer from the side of medium voltage(MV) bushings whereas the wall visible at the right side of Fig. 3 ("positive values" of distances from the center of the tap changer) is the wall from the side of high voltage (HV) bushings. The wall with the tap changer is presented at the center of the figure. The areas marked by the most intensive color and greatest values of descriptors $(-3$ and -2$)$ are areas where advanced degree of AE signals is the highest. Basing on the made analysis, one can state that there are at least two particular danger PD sources within the tested transformer. One source (coordinates -200 and +150$)$ is the source placed in winding insulation system of L1 phase. Such an AE analysis result was quiet convergent with the result coming from measurements carried out by means of electric method. $\mathrm{A}$ riddle was the source located by $\mathrm{AE}$ method at the point with coordinates +110 and +50 . It needs to remind that result obtained from electric method testifies that there is PD source connected with an insulation system of L3 phase, whereas analysis result was location of the source near L1 phase. Discrepancy of results coming from two methods ceased to be incomprehensible after revision of the insulation system of T1 transformer and looking at construction details of the transformer whose winding arrangement of particular phases with tap changer is as in Fig. 4.

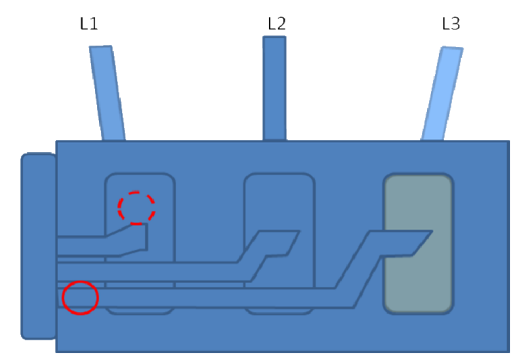

Fig. 4. Winding phase arrangements of T1 transformer with the tap changer and the place in the transformer when PD sources occur.

The second PD source in analyzed transformer was situated within insulation of conductors connecting the winding of L3 phase with the tap changer.

As a summing-up of analysis relating to insulation system of $\mathrm{T} 1$ transformer one can state that there are at least two danger PD sources: the first placed within insulation of conductors connecting L3 phase winding with the tap charger (near this tap, see full red circle) and the second placed within insulation of L1 phase (at place marked in Fig. 4 intermittent red circle).

The second of tested transformers was a new unity (Fig. 5), which has been stated previously.

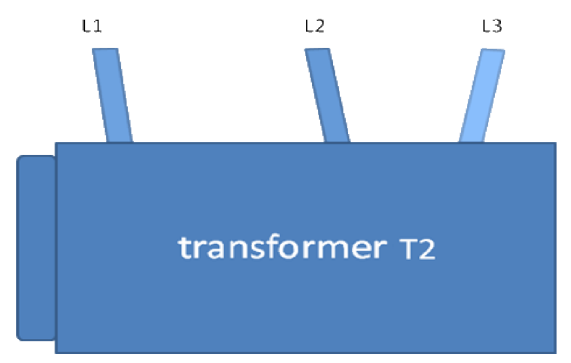

Fig. 5. General view of T2 transformer (25 MVA, $220 \mathrm{kV})$.

Basing on measurements made by electric method at $1.7 U_{\mathrm{r}}$, according to recommendations of PN-EN 60270 standard, one can state surface discharges with apparent charge of $1 \mathrm{nC}$ situated near L2 phase. That was a reason to verify the insulation system of T2 transformer using combined method of PD measurements, proposed by the 
authors of the article. Result of measurements made by means of $\mathrm{AE}$ is shown at the map of ADC descriptors (Fig. 6).

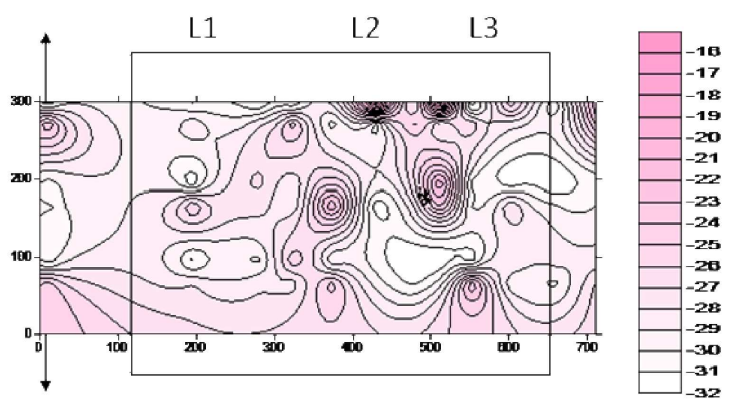

Fig. 6. Map of ADC descriptors worked up within the band of $80-180 \mathrm{kHz}$ after analysis of AE signals recorded in T2 transformer.

It is readily noticeable that in such a case none of the danger sources was detected. At most, there are only areas with little great descriptor values between L2 and L3 phases at upper part of transformer vat. Results coming from electric method, obtained by unconventional way, prove - as in the case of $\mathrm{T} 1$ transformer - such a verdict (Fig. 7).

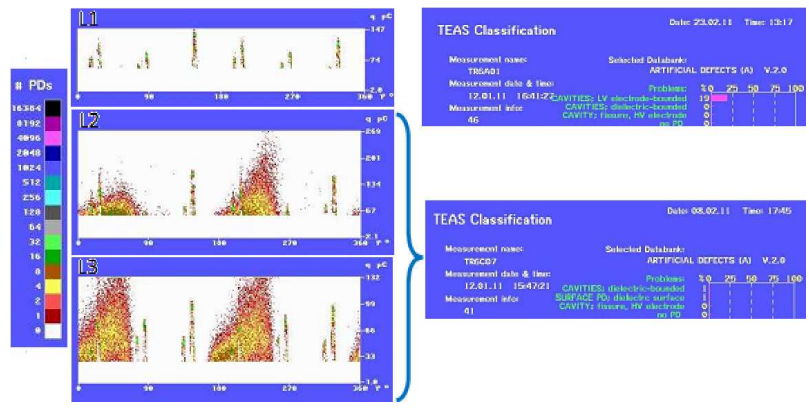

Fig. 7. PD phase pictures and effect resulting from TEAS diagnostic program for L1, L2 and L3 phases of $\mathrm{T} 2$ transformer.

None of PD sources were detected in the case of L1 phase. Weak sources of surface charges have been stated in L2 and L3 phases, but levels of apparent charge had 150 and $100 \mathrm{pC}$, respectively, allowable for the insulation system of the transformer. The conclusion was as follows: defect of insulation system stated previously was not preventing transformer operation at the voltage not higher than allowable one for this unity. However, taking into account overvoltages, additional measuring experiment was carried out. It consisted in use of AE method realized in untypical way - when only one phase of the transformer was energized by means of voltage of $1.7 U_{\mathrm{r}}$ (by consent of the owner) by at will period of time. Result of this measurement was rather surprising. None PD sources were noted by first $1.5 \mathrm{~h}$ but - after - a lot of sources, filling almost full vat of the transformer, appeared (Fig. 8).

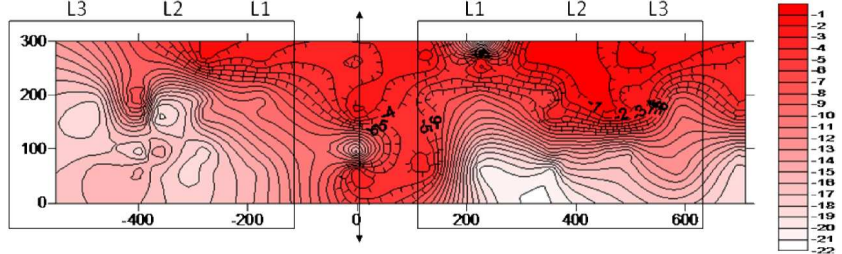

Fig. 8. Map of ADC descriptors worked up within the band of $80-180 \mathrm{kHz}$ after analysis of AE signals recorded in $\mathrm{T} 2$ transformer after $1.5 \mathrm{~h}$ of supply by $1.7 U_{\mathrm{r}}$ voltage.

Such a great number of PD sources was a result of "gassing" of the oil filling transformer vat, phenomenon rather common during enlarged voltage or long over voltages. Revision of the insulation system of T2 transformer proved existence of small conducting track on the surface of solid dielectric.

\section{Conclusions}

Diagnostic tests of transformers should be as more fully (more numerous) as much is technical importance of a given unity. Transformer diagnosis is an activity very composed, based on simultaneous evaluation of values of different partial quantities, frequently opposed mutually. Combined method enables us to record simultaneously acoustic, electric and chemical "activity" of partial discharges within insulation systems of the transformer, and may be one of important diagnostic methods at test stations and - in the future - also at the place of transformer operation.

\section{Acknowledgments}

The researches were financed in the frame of the project N N511 458836 of the Ministry of Science and Higher Education.

\section{References}

[1] J. Singh, Y.R. Sood, R.K. Jarial, P. Verma, IEEE Electr. Insulat. Mag. 24, 11 (2008).

[2] Framework Operation Instruction of Transformers, Energopomiar-Elektryka, Gliwice 2001 (in Polish).

[3] F.H. Krueger, E. Gulski, A. Krivda, IEEE Trans. Electr. Insulat. 28, 917 (1993).

[4] P. Bartnikas, IEEE Trans. Diel. Electr. Insul. 9, 763 (2002).

[5] B. Florkowska, M. Florkowski, R. Włodek, P. Zydroń, Mechanisms, Measurements and Analysis of Partial Discharges in Diagnose of High Voltage Insulating Systems, IPPT PAN, Warsaw 2001 (in Polish).

[6] F. Witos, Investigation of Partial Discharges by Means of Acoustic Emission Method and Electric Method, Wydawnictwo Politechniki Śląskiej, Gliwice 2008 (monograph in Polish). 
[7] D. Duda, Z. Gacek, M. Szadkowski, Computer Aided Analysis of Partial Discharges. Computer Application in Electrical Engineering (monograph), Publ. of IEP Poznań, 2004, p. 228.

[8] L.E. Lundgaard, IEEE Electr. Insulat. Mag. 8, 34 (1992).

[9] T.F. Drouillard, J. Acoust. Emission 14, 1 (1996).

[10] J. Skubis, Acoustic Emission within Investigation of Dielectric Insulations and Power Installations, IPPT PAN, Warsaw 1993 (in Polish).

[11] J. Skubis, State of Elaboration of AE Method in WSI Opole and Directions of Its Development, ZN WSI Opole, No. 184, Elektryka z. 36, Opole 1992, p. 5 (in Polish).

[12] M. MacAlpine, Z. Zhiquiang, M.S. Demokan, Electric Power Syst. Res. 63, 27 (2002).
[13] J. Deng, H. Xiao, W. Huo, M. Luo, R.G. May, A. Wang, Y. Liu, Opt. Laser Technol. 33, 305 (2001).

[14] T. Boczar, IEEE Trans. Diel. Electr. Insulat. 8, 598 (2001).

[15] M. Duval, J. Dukarm, IEEE Elect. Insul. Mag. 21, 21 (2005).

[16] F. Witos, Z. Gacek, Z. Opilski, Mol. Quant. Acoust. 24, 237 (2003).

[17] F. Witos, Z. Gacek, Europ. Phys. J. ST 154, 239 (2008).

[18] F. Witos, Z. Gacek, Acta Phys. Pol. A 116, 422 (2009).

[19] F. Witos, A. Olszewska, Acta Phys. Pol. A 118, 1267 (2010). 